\section{Artritis infecciosa de la articulación temporomandibular consecutivo a otitis necrotizante: reporte de un caso}

\author{
FERNANDA PINTOR $^{1, \mathrm{a}}$, ROLANDO CARRASCO ${ }^{2, \mathrm{~b}}$, \\ FRANCISCO VERDUGO-AVELLO ${ }^{3, \mathrm{c}}$, MIRTHA LANDAETA ${ }^{4, \mathrm{~d}}$
}

\section{Septic arthritis of the temporomandibular joint. Report of one case}

Septic arthritis of the temporomandibular joint (TMJ) is an uncommon condition, caused by hematic bacterial migration or direct migration of other head and neck infections. We report a 41 year old female who presented a right temporomandibular joint involvement, with bone destruction of the mandibular condyle and an infectious process spreading to the temporal space, following a necrotizing medial and external otitis with associated mastoiditis. A septic arthritis of the TMJ by continuity was diagnosed and treated with antimicrobials, TMJ arthrocentesis and occlusal stabilization, with a positive evolution. However, the patient remains in control due a secondary TMJ osteoarthritis caused by the septic arthritis.

(Rev Med Chile 2015; 143: 805-808)

Key words: Anti-bacterial agents; Arthritis, infectious; Temporomandibular joint.
${ }^{1 a}$ Cirujano Maxilofacial, Servicio de Cirugía Maxilofacial, Hospital San Juan de Dios, Hospital Clínico de la Universidad de Chile,

Santiago, Chile.

${ }^{2 b}$ Becario Cirugía Maxilofacial Universidad de Chile, Servicio de Cirugía Maxilofacial Hospital San Juan de Dios, Santiago, Chile. ${ }^{3 c}$ Cirujano Dentista, Santiago, Chile.

${ }^{4 \mathrm{~d} C i r u j a n o ~ M a x i l o f a c i a l, ~ S e r v i c i o ~}$ de Cirugía Maxilofacial, Hospital San Juan de Dios, Santiago, Chile.

Recibido el 31 de diciembre de 2014, aceptado el 29 de marzo de 2015.

Correspondencia a:

Fernanda Pintor

Teléfono: 992265187

fernandapintor@gmail.com
L a artritis séptica o infecciosa es un proceso inflamatorio agudo producido por la invasión y multiplicación de microorganismos piógenos dentro de una articulación. Se caracteriza por presentar signos y síntomas como dolor, fiebre, edema y pérdida de función de una o más articulaciones ${ }^{1}$. Puede generar destrucción importante de la articulación comprometida llegando incluso a la pérdida funcional irreversible ${ }^{2}$. Es más común en rodillas y caderas ${ }^{3,4}$.

La artritis infecciosa de la articulación temporomandibular (ATM) es una enfermedad poco común, con sólo 40 casos reportados en los últimos 70 años ${ }^{5}$. La causa sería la migración bacteriana por vía hematógena o por infección continua directa ${ }^{2}$. Se han reportado factores predisponentes locales como procesos infecciosos en sitios cercanos como la otitis media, trauma penetrante, quemaduras e iatrogenia ${ }^{6,7,8}$. Dentro de los factores generales o sistémicos, encontramos cuadros como la artritis reumatoide, diabetes mellitus, inmunosupresión, uso prolongado de esteroides y algunas enfermedades de transmisión sexual como la gonorrea y sífilis ${ }^{9,10}$. Los patógenos más comúnmente encontrados son el Staphylococcus aureus, Neisseria, Haemophilus influenzae y estreptococos ${ }^{2,11,12}$.

El objetivo de este artículo es reportar el caso de una paciente con un cuadro de artritis infecciosa de la ATM consecutivo a un proceso infeccioso vecino de origen ótico, haciéndose énfasis en la relevancia del diagnóstico precoz, tratamiento y manejo de las secuelas.

\section{Reporte del caso}

Paciente femenino de 41 años, con antecedente de Diabetes Mellitus tipo 2 tratada farmacológicamente. Ingresa al servicio de otorrinolaringología (ORL) del Hospital San Juan de Dios por un cuadro de otalgia derecha de 3 meses de evolución, de 
inicio insidioso, el cual aumenta progresivamente llegando a una intensidad 10/10 al momento de la consulta, según la Escala Visual Análoga (EVA). Al examen la paciente se encontraba afebril, sin embargo se evidencia otorrea purulenta, hipoacusia y aumento de volumen preauricular ipsilateral. El estudio del cuadro arroja como diagnóstico una otitis externa necrotizante, además de una mastoiditis ebúrnea. El manejo inicial del cuadro consistió en la hospitalización y el tratamiento antibiótico con un esquema empírico enfocado en el proceso ótico con ceftazidima más clindamicina endovenosa y ciprofloxacino tópico.

El grado de compromiso es objetivado en las imágenes de la tomografía axial computarizada (TAC) que muestra una franca zona de erosión de la superficie ósea condilar mandibular derecha, con pérdida de capa cortical y destrucción hasta la medular ósea (Figura 1). Por lo anterior se interconsulta a cirugía maxilofacial. Al examen se aprecia el aumento de volumen preauricular derecho de límites difusos, consistencia firme, con aumento de temperatura local y eritema asociados. El examen funcional revela disminución considerable de la apertura bucal de $20 \mathrm{~mm}$ aproximadamente con movimientos de lateralidad conservados, asociándose a artralgia exacerbada por la función. Al examen hay ausencia de múltiples piezas dentarias posteriores tanto superiores como inferiores. La resonancia nuclear magnética (RNM) muestra cambios degenerativos y erosión a nivel cortical de la vertiente anterior del cóndilo mandibular además de un proceso inflamatorio intracapsular diseminado a los músculos masticadores y al espacio temporal del mismo lado (Figura 2). Con los hallazgos clínicos e imagenológicos se plantea la hipótesis diagnóstica de artritis infecciosa de la ATM por continuidad.

Luego de establecido el diagnóstico de otitis necrotizante y mastoiditis, es ingresada a pabellón por parte del equipo de ORL para realizar una mastoidectomía radical modificada. Posteriormente se realiza una punción intraarticular para estudio del contenido, donde se aprecia salida de contenido líquido turbio y seropurulento que se envía a cultivo bacteriológico, el cual resulta negativo a las $72 \mathrm{~h}$ de incubación. Se realiza además una artroscentesis con doble aguja de la ATM según la técnica de Nitzan et $a^{13}$, realizando un lavado profuso de la articulación con solución salina. La paciente posterior a estas intervenciones mejora su condición general, siendo dada de alta con disminución franca de la sintomatología, del aumento de volumen y una mejoría de la apertura bucal llegando hasta los $40 \mathrm{~mm}$.

$\mathrm{Al}$ control al mes, se presenta asintomática. Sin embargo, se aprecia la presencia de un ruido articular en apertura y cierre el cual evoluciona a crépito en los siguientes controles. Es derivada a tratamiento protésico para mejorar la estabilidad oclusal y por ende disminuir la sobrecarga articu-

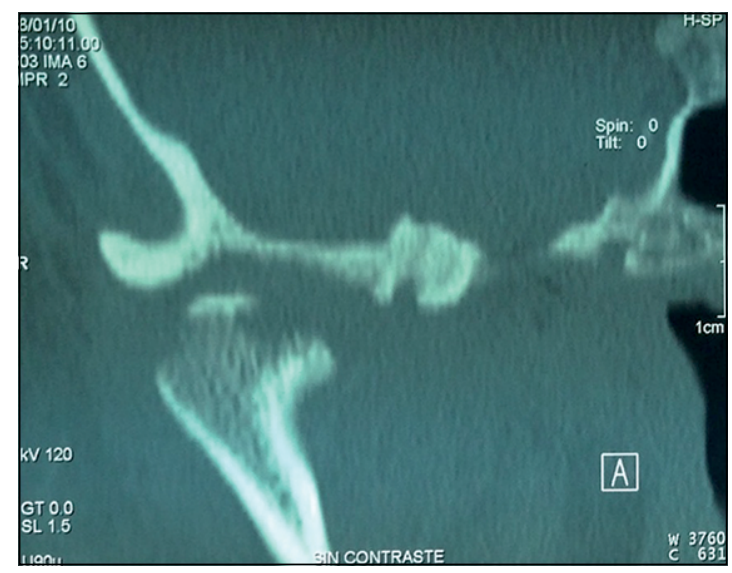

Figura 1. Erosión de la superficie ósea condilar mandibular derecha, con pérdida de cortical y destrucción hasta la medular ósea en TAC.

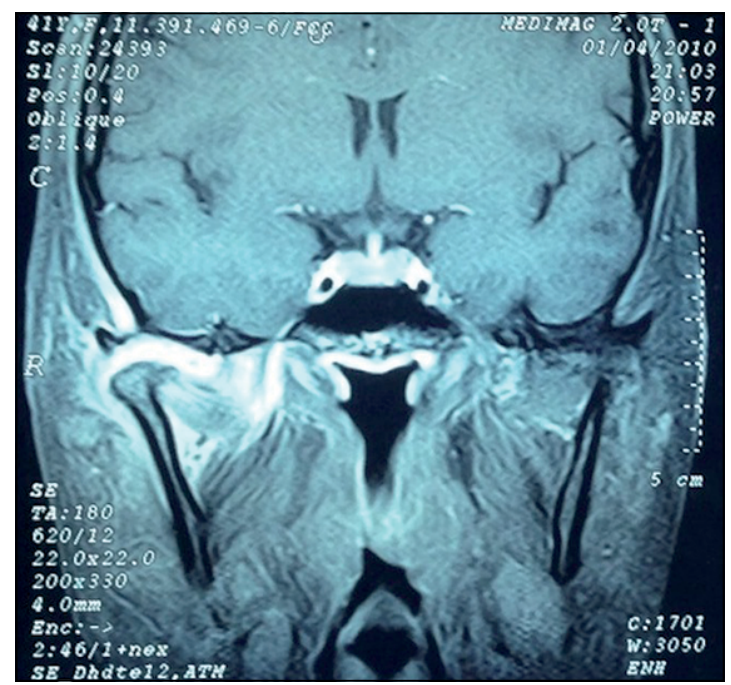

Figura 2. Proceso inflamatorio intracapsular diseminado a músculos masticadores y al espacio temporal del mismo lado en RNM. 
lar. Se pide TAC de control a los 2 meses el cual confirma una regeneración parcial de la cortical ósea condilar. Actualmente, cuatro años después, se encuentra asintomática, con dinámica mandibular conservada. Sin embargo, como secuela de la otitis media y posterior mastoiditis queda con alteraciones auditivas a la percepción de ruidos graves además de la persistencia de crépito de la ATM derecha debido al daño irreversible de los tejidos intraarticulares.

\section{Discusión}

La artritis séptica es un cuadro poco común en la ATM, cuya etiología proviene de una extensión directa de otra infección de cabeza y cuello; sin embargo, estudios recientes de la ATM y de otras articulaciones demostraron que los microorganismos son más comúnmente implantados de forma hematógena ${ }^{14}$. En este caso la infección articular fue provocada por continuidad del cuadro de otitis externa necrotizante y la consecutiva mastoiditis aguda. Esta última se considera la complicación extracraneal más frecuente de la otitis supurativa ${ }^{14}$, la cual se propaga a partir de las celdas mastoídeas, exteriorizándose hacia el periostio subyacente ${ }^{15}$. Una vez instalada la artritis séptica, se manifiesta clínicamente con compromiso unilateral de la articulación, dolor preauricular espontáneo, fiebre y eritema de la zona. El edema preauricular es una manifestación menos frecuente ${ }^{15,16}$. El diagnóstico incluye el reconocimiento de las manifestaciones clínicas, exámenes de laboratorio, hallazgos imagenológicos, y en lo posible el análisis del líquido intrarticular ${ }^{17}$. Se debe sospechar en pacientes que debutan con dolor severo y repentino de la ATM, trismus y maloclusión aguda ${ }^{5}$.

Las imágenes complementarias constituyen una importante ayuda para el diagnóstico. En este caso, al contrario de lo descrito en la literatura, donde se establece que los cambios degenerativos óseos son raros en las fases agudas ${ }^{16}$ fue uno de los primeros elementos que sugirieron que se trataba de una artritis séptica debido a que las manifestaciones clínicas tanto de la otitis como de la mastoiditis impedían el reconocimiento del cuadro articular conjunto. La RNM es altamente recomendada ya que su sensibilidad permite una rápida detección de la diseminación. Además permite visualizar los tejidos blandos adyacentes, como en este caso, evidenciando el compromiso muscular y del espacio temporal.

Idealmente se debe realizar una aspiración para cultivo $^{14}$. En cuanto a los resultados negativos al cultivo, se pueden explicar por la antibioterapia empírica instaurada previamente, lo cual probablemente modificó la flora al momento de la aspiración o por problemas en el traslado y cultivo bacteriano. Sin embargo, el resultado negativo de presencia bacteriana en la sinovial no parece ser un evento aislado en este cuadro ${ }^{5}$.

Debido a los pocos casos reportados, aún no hay consenso en cuanto al tratamiento de la artritis infecciosa de la ATM. El tratamiento antibiótico, drenaje e inmovilización son algunos de los componentes considerados claves hasta ahora para el tratamiento de esta patología ${ }^{17}$. En la fase aguda, la antibioterapia empírica se debe instaurar lo más precoz posible, con antibióticos de amplio espectro para luego, con el cultivo y antibiograma, comenzar la terapia específica. En este caso, debido a la correcta evolución de la paciente con la terapia instaurada por el equipo de ORL, esta no fue modificada ${ }^{2}$. El segundo componente terapéutico es el lavado intraarticular, lo que en este caso fue realizado mediante una artroscentesis ${ }^{13}$. Junto a esto, fue indicada la confección de una prótesis parcial removible y un plano estabilizador nocturno, para mejorar la inestabilidad oclusal preexistente derivada de la pérdida de la mesa oclusal posterior. El uso de férulas y dispositivos interoclusales ha sido descrito y forma parte del esquema de algunos autores para el tratamiento de esta patología ${ }^{17}$. También se han reportado como terapia coadyuvante la artroscopia de la ATM, siendo reservada para casos donde la artrocentesis ha sido inefectiva ${ }^{18}$. En este caso la antibioterapia en conjunto con la artrocentesis y la terapia funcional oclusal dio buenos resultados, por lo que concluimos que una buena función y estabilidad oclusal son factores coadyuvantes relevantes en el tratamiento de esta patología.

Es importante destacar que las secuelas anatómicas intraraticulares irreversibles condicionan un cuadro de artrosis secundaria, el cual debe ser controlado y manejado en el tiempo. El manejo terapéutico de la artrosis temporomandibular tanto primaria como secundaria puede ir desde procedimientos no invasivos como el uso de AINES, relajantes musculares y férulas oclusales, hasta procedimientos quirúrgicos invasivos como 
reconstrucciones parciales de la ATM o recambios articulares totales dependiendo de la sintomatología que produzcan en el paciente ${ }^{19}$.

El tiempo del diagnóstico y del tratamiento son esenciales para limitar posibles secuelas y complicaciones consecutivas a una artritis séptica, como la diseminación de la infección, disfunción articular, trastornos del crecimiento, fibrosis y anquilosis de la $\mathrm{ATM}^{20}$.

\section{Referencias}

1. Morrissy R. Bone and joint infections. En: Morrissy R, Pediatric Orthopaedics. Philadelphia, editorial Lovell and Winter's 1990; p 539.

2. O’Meara P, Bartal E. Septic arthritis: process, etiology, treatment outcome: a literature review. Orthopedics 1988; 11 (4): 623-8.

3. Nade S. Acute septic arthritis in infancy and children. J Bone Joint Surg Br 1983; 65B (3): 234-41.

4. Studahl M, Bergman B, Kälebo P, Lindberg J. Septic arthritis of the knee: A 10-year review and long-term follow-up using a new scoring system. Scand J Infect Dis 1994; 26 (1): 85-93.

5. Cai XY, Yang C, Zhang ZY, Qiu WL, Chen MJ, Zhang SY. Septic arthritis of the temporomandibular joint: a retrospective review of 40 cases. J Oral Maxillofac Surg 2010; 68 (4): 731-8.

6. Thomson H. Septic arthritis of the temporomandibular joint complicating otitis externa. J Laryngol Otol 1989; 103 (3): 319-21.

7. McCain J, Zabiegalski N, Levine R. Joint infection as a complication of temporomandibular joint arthroscopy: a case report. J Oral Maxillofac Surg 1993; 51 (12): 138992.

8. Hilbert L, Peters W, Tepperman P. Temporomandibular joint destruction after a burn. Burns Incl Therm Inj 1984; 10 (3): 214-6.

9. Trimble L, Schoenaers J, Stoelinga P. Acute suppurative arthritis of the temporomandibular joint in a patient with rheumatoid arthritis. J Oral Maxillofac Surg 1983; 11 (2): 92-5.

10. Goodman W, Strelzow V. Infections of the temporomandibular joint. J Otolaryngol 1979; 8 (3): 250-4.

11. Borenstein D, Simon G. Haemophilus influenzae septic arthritis in adults. A report of four cases and a review of the literature Medicine. 1986; 65 (3): 191-201.

12. Deesomchok U, Tumrasvin T. Clinical study of culture-proven cases of non-gonococcal arthritis. J Med Assoc Thai 1990; 73 (11): 615-23.

13. Nitzan D, Dolwick M, Martínez G. Temporomandibular joint arthrocentesis: a simplified treatment for severe, limited mouth opening. J Oral Maxillofac Surg 1991; 49 (11): 1163-7.

14. Goldschmidt M, Butterfield K, Goracy E, Goldberg M. Streptococcal infection of the temporomandibular joint of hematogenous origin: A case report and contemporary therapy. J Oral Maxillofac Surg 2002; 60 (11): 1347-53.

15. Bakhos D, Trijolet J, Morinieri S, Pondaven S, Al Zahrani M, Lescanne E. Conservative management of acute mastoiditis in children. Arch Otolaryngol Head Neck Surg 2011; 137 (4): 346-50.

16. Atsu S, Ayhan-Ardic F. Temporomandibular disorders seen in rheumatology practices: a review. Rheumatol Int 2006; 26 (9): 781-7.

17. Valmaseda E, Escoda C. Diagnóstico y tratamiento de la patología de la articulación temporomandibular ORLDIPS 2002; 29 (2): 55-70.

18. Murakami K, Matsumoto K, Iizuka T. Suppurative arthritis of the temporomandibular joint. Report of a case with special reference to arthroscopic observations. J Maxillofac Surg 1984; 12 (1): 41-5.

19. Mercuri L. Osteoarthritis, Osteoarthrosis, and Idiopathic Condylar Resorption. Oral Maxillofacial Surg Clin N Am 2008; 20 (2): 169-83.

20. Sembronio S, Albiero A, Robiony M, Costa F, Toro C, Polit M. Septic arthritis of the temporomandibular joint successfully treated with arthroscopic lysis and lavage: case report and review of the literatura. Oral Surg Oral Med Oral Pathol Oral Radiol Endod 2007; 103 (2): 1-6. 